\title{
Atomic number fluctuations in a falling cold atom cloud
}

\author{
Astrid Lambrecht, Elisabeth Giacobino and Serge Reynaud \\ Laboratoire Kastler Brossel, UPMC, ENS, CNRS \\ Université Pierre et Marie Curie, case 74, F-75252 Paris, France \\ (Quantum Semiclassical Optics 8, 457 (1996))
}

\begin{abstract}
We evaluate the effective number of atoms in experiments where a probe laser beam with a Gaussian transverse profile passes through an atomic medium consisting in a cold atom cloud released from a magnetooptical trap. Considering the case where the initial distribution is a Gaussian function of position and of velocity, we give a quantitative description of the temporal variation of the effective number while the cloud is exploding and falling down. We discuss the two cases where the effective number is defined from the linear and nonlinear phaseshifts respectively. We also evaluate the fluctuations of the effective atomic number by calculating their correlation functions and the associated noise spectra. We finally estimate the effect of these fluctuations on experiments where the probe beam passes through a cavity containing the atomic cloud.
\end{abstract}

\section{Introduction}

In recent years, the development of magneto-optical traps [1] has opened the way to the use of cold atoms for various purposes such as atomic interferometry, and nonlinear or quantum optics. In many of these experiments, a cloud of cold atoms is first trapped and then released when the laser beams and the magnetic field of the trap are turned off. The experiment is thus performed while the cloud is undergoing a ballistic expansion due to the velocity distribution in the trap and falling down under the influence of gravity. Models using a Monte-Carlo simulation of the evolution of the atomic positions and velocities have been developed to analyze the free fall of the cloud of cold atoms. They have been used to obtain informations about the initial position and velocity distribution in the trap from fluorescence measurements, particularly to evaluate temperature in the trap when it is released and to investigate deviations from a Maxwellian velocity distribution ([2] and references therein).

Here, we want to concentrate on the category of experiments where the falling cloud of cold atoms is used in spectroscopy, nonlinear or quantum optics experiments. In this case, the cloud interacts with a probe laser beam that passes through it. A crucial point for these experiments lies in the definition 
of an effective number of atoms contributing to the interaction with the probe beam and also to variation of this effective atomic number as a function of time.

A first aim of the present paper is to provide a definition of the effective number of atoms in the probe beam, as well as a precise modelisation of its temporal evolution. We will show that this is a quite involved problem in the general case, and in particular that different definitions may be given, depending upon the specific physical effect chosen as a reference for the evaluation of the atomic number. We will discuss the example where the linear phase produced by the atomic medium is chosen as a reference, as well as the case where the nonlinear phase shift is considered. This general discussion will lead to simpler conclusions in the two limiting cases where either the transverse size of the probe beam is much smaller than the cloud size or the Rayleigh length characteristic of the divergence of the probe beam is much larger than the cloud size.

A further problem is that this effective number of atoms has fluctuations. These fluctuations must be added to the fluctuations usually evaluated with the number of atoms kept constant and they may have an influence in spectroscopy, metrology or quantum optics experiments. In this class of experiments indeed, the signal is often measured with such a high precision that any excess noise produced by the atomic medium can appear as a limiting factor for the sensitivity.

This is why in a second part of this paper we will evaluate atomic number fluctuations by calculating their characteristic correlation functions. We will show in particular that it is possible to calculate time dependent noise spectra, when the transverse size of the probe beam is much smaller than the cloud size. In this case indeed, the correlation time, expected to be of the order of the time of flight through the probe beam, is much smaller than the typical variation time of the mean number of atoms, of the order of the time of flight through the cloud, so that the effective atomic number may be considered as a quasistationary random variable.

The theoretical developments of the present paper are in particular essential for the understanding and interpretation of experimental results we obtained recently [3]. The experiment consists in the measurement of quantum fluctuations of a weak probe beam passing through a cloud of cold atoms expanding and falling down after it has been released from a standard magneto-optical trap. To enhance the interaction between atoms and light, the atoms are placed inside an optical cavity. The variation of the number of atoms leads to a continuous variation of the linear phaseshift of the laser beam, which is sufficient to scan the cavity across resonance. Due to the non-stationary character of the measurement of quantum fluctuations, the knowledge of the time variation of the number of atoms plays a crucial role in the interpretation of experimental results. The fit with the experimental results, using the theory developed in the present paper, will be presented in a further publication 顿. While a variation of the mean atomic number scans the cavity detuning through a variation of the linear phase shift, atomic number fluctuations result in fluctuations of the 
detuning which have an effect on the fluctuations of the probe beam which are measured in the experiment. If they are large enough, they may enter exponentially in the expression for the field fluctuations after interaction, and therefore produce excess noise not only at the frequencies contained in their own noise spectrum, but also at higher frequencies through a multiplicative noise processing [5]. The evaluation of atomic number fluctuations contained in the present paper are particularly useful to delineate under which conditions these fluctuations degrade squeezing measurements.

As already pointed out by Weiss et al. [6], the density distribution of a freely falling atom cloud can be calculated analytically at any instant, if a Gaussian profile in the trap is assumed for position density as well as for velocity distribution. Experiments by Drewsen et al. [2] have shown this assumption to be justified if multiple scattering effects become negligeable, that means for large clouds and atoms with a temperature in the $100 \mu \mathrm{K}$ to $\mathrm{mK}$ range [7]. These conditions are met in the experiment that we are modelizing [3]. When the number of trapped atoms is deduced from their fluorescence signal, the detectivity function does not have a Gaussian profile and does in general not lead to analytical results. In the case studied in this paper, the experimental determination of the number of atoms relies on a phase shift measurement of a probe laser beam. It may thus be expected that the detectivity function is given by the Gaussian profile of the laser beam, leading to analytical solutions for the mean atomic number and for correlation functions of atomic number fluctuations.

In Section 2 of this paper, we describe our model in more detail and show how the effective number of atoms is deduced from phase shift measurements, accounting for the transverse mode structure of the probe beam. From this, we analyze in Section 3 the expression of the case of a linear phase shift measurement, considering the general case of an atomic motion under gravity. In Section 4 , we address the problem of a saturated phase shift. The correlation functions and the noise spectrum of atomic number fluctuations are then calculated in Section 5 . When evaluating fluctuations, we restrict our attention to the particular case of the atomic number deduced from the linear phase shift since we are mainly interested in potential effects of linear phase fluctuations. We finally give an estimate of the effect of the latter on the probe-cavity detuning in Section 6 .

\section{Description of the model}

As already discussed, we consider that the number of atoms is monitored through a phase measurement performed on a probe laser beam. Hence, this number will be obtained as an integral of the atomic density over the volume, with a weight function fitting the Gaussian profile of the laser beam.

To determine precisely this weight function, we introduce the Gaussian mode $u(\mathbf{r})$ ( $\mathbf{r}$ stands for the three-dimensional position $(x, y, z))$ describing the intra- 
cavity field propagating along the $x$ direction and coming onto the atoms:

$$
u(\mathbf{r})=\sqrt{\frac{2}{\pi}} \frac{1}{w(x)} \exp \left[-\frac{y^{2}+z^{2}}{w^{2}(x)}-i \varphi(\mathbf{r})\right]
$$

$w(x)$ is the position-dependent beam size:

$$
\begin{gathered}
w^{2}(x)=w_{0}^{2}\left(1+\frac{x^{2}}{l_{R}^{2}}\right) \\
l_{R}=\frac{\pi w_{0}^{2}}{\lambda}
\end{gathered}
$$

where $w_{0}$ is the beam waist, $l_{R}$ the Rayleigh divergence length and $\lambda$ the laser wavelength. The field phase $\varphi(\mathbf{r})$ is the sum of contributions representing propagation phase and curvature of the wave at longitudinal position $x$ :

$$
\varphi(\mathbf{r})=-\frac{2 \pi x}{\lambda}+\arctan \left(\frac{x}{l_{R}}\right)-\frac{\pi}{\lambda} \frac{\left(y^{2}+z^{2}\right) x}{x^{2}+l_{R}^{2}}
$$

The squared modulus of the Gaussian mode:

$$
|u(\mathbf{r})|^{2}=\frac{f(\mathbf{r})}{S(x)}
$$

may be written in terms of a Gaussian weight function $f(\mathbf{r})$ normalized to unity on the beam axis and an effective beam section $S$ :

$$
\begin{gathered}
f(\mathbf{r})=\exp \left[\frac{-2\left(y^{2}+z^{2}\right)}{w^{2}(x)}\right] \\
S(x)=\frac{\pi w^{2}(x)}{2}
\end{gathered}
$$

The normalisation of the Gaussian mode $u(\mathbf{r})$ has been chosen so that:

$$
\int|u(\mathbf{r})|^{2} d y d z=1
$$

This relation holds for any position $x$ along the propagation axis.

We will write the incident field seen at time $t$ by the atoms located at point $\mathbf{r}$ in the interaction representation with respect to the laser frequency $\omega_{L}$ :

$$
E(\mathbf{r}, t)=e^{-i \omega_{L} t} E(\mathbf{r}, t)+e^{i \omega_{L} t} E(\mathbf{r}, t)^{\dagger}
$$

where $E(\mathbf{r}, t)$ is the product of the Gaussian profile $u(\mathbf{r})$ by a mode amplitude $A(t)$ :

$$
E(\mathbf{r}, t)=\sqrt{\frac{\hbar \omega_{L}}{2 \varepsilon_{0} c}} A(t) u(\mathbf{r})
$$


This definition is such that $A(t)^{\dagger} A(t)$ is the number of photons passing through a beam section per unit time.

As explained below, the field radiated by the atoms will have a different transverse profile as a consequence of the nonlinear character of light-matter interaction. The Gaussian mode $u(\mathbf{r})$ is the lowest order mode $u_{00}$ of the family of orthogonal Hermite modes. We will consider that the higher-order Hermite modes $u_{m n}$ (with $m \neq 0$ or $n \neq 0$ ) are not resonant at the same frequency as the lowest order mode $u_{00}$. Hence, any field radiated by the atoms into these higher-order modes will not be efficiently coupled back onto the atoms and can therefore be disregarded. We will then calculate the field amplitude radiated into the fundamental mode by a mere projection.

The effect of an optically thin atomic layer of length $d x$ may be described by a local modification $d E(\mathbf{r})$ of the field:

$$
E(\mathbf{r}) \rightarrow E(\mathbf{r})+d E(\mathbf{r})
$$

with [8]:

$$
d E(\mathbf{r})=-\frac{3 \lambda^{2}}{4 \pi} \rho(\mathbf{r}) \alpha(\mathbf{r}) E(\mathbf{r}) d x
$$

Note that we have treated the motion of atoms as quasistatic and neglected any consequence of the Doppler effect. As a result, we have used the atomic density $\rho(\mathbf{r})$ integrated over the velocity distribution. $\alpha(\mathbf{r})$ is the atomic polarizability measured as a dimensionless number:

$$
\alpha(\mathbf{r})=\frac{\alpha_{l}}{1+2 s(\mathbf{r})}
$$

The linear polarizability $\alpha_{l}$ is a function of the dimensionless detuning $\delta$ between laser frequency $\omega_{L}$ and atomic resonance frequency $\omega_{0}$ normalized to the decay rate $\gamma$ of the atomic dipole:

$$
\begin{gathered}
\alpha_{l}=\frac{1}{1+i \delta} \\
\delta=\frac{\omega_{0}-\omega_{L}}{\gamma}
\end{gathered}
$$

The saturation parameter $s$ is proportional to the local laser intensity $|\beta(\mathbf{r})|^{2}$ measured as a dimensionless number:

$$
\begin{gathered}
s(\mathbf{r})=\frac{|\beta(\mathbf{r})|^{2}}{1+\delta^{2}} \\
\beta(\mathbf{r})=\frac{d_{0} E(\mathbf{r})}{\hbar \gamma}=\sqrt{\frac{3 \lambda^{2}}{4 \pi}} u(\mathbf{r}) \frac{A}{\sqrt{\gamma}}
\end{gathered}
$$


where $d_{0}$ is the matrix element of the atomic dipole.

As a first step, we consider that the saturation of polarizability may be neglected; we will come back to the nonlinear case later on. The number of atoms is then evaluated by monitoring the linear phase shift produced by the atomic cloud, with $d E(\mathbf{r})$ written as:

$$
d E(\mathbf{r})=-\frac{3 \lambda^{2}}{4 \pi} \rho(\mathbf{r}) \alpha_{l} E(\mathbf{r}) d x
$$

Even in this linear case, the radiated field $d E(\mathbf{r})$ does not have the same spatial variation as the incident field $E(\mathbf{r})$ when the atomic density $\rho(\mathbf{r})$ is positiondependent. Ignoring the field radiated into higher-order modes, one gets the modification of the field amplitude $d A$ in the Gaussian mode by projecting $d E(\mathbf{r})$ onto $u(\mathbf{r})$ :

$$
\sqrt{\frac{\hbar \omega_{L}}{2 \varepsilon_{0} c}} d A=\int d E(\mathbf{r}) u^{*}(\mathbf{r}) d y d z
$$

Assuming that the modification of the field by the whole atomic cloud remains small (assumption of an optically thin atomic cloud), we obtain this modification by summing up the contributions of all the atomic layers as:

$$
\frac{d A}{A}=-\frac{3 \lambda^{2}}{4 \pi} \alpha_{l} \sigma
$$

with :

$$
\sigma=\int|u(\mathbf{r})|^{2} \rho(\mathbf{r}) d \mathbf{r}
$$

The modification of the field intensity is:

$$
d\left(A^{\dagger} A\right)=A^{\dagger} d A+\left(d A^{\dagger}\right) A=-A^{\dagger} A \frac{3 \lambda^{2}}{2 \pi} \frac{\sigma}{1+\delta^{2}}
$$

As the absorption cross section is $\left(3 \lambda^{2} / 2 \pi\right)$ at resonance and is divided by $\left(1+\delta^{2}\right)$ otherwise, this equation shows that $\sigma$ must be interpreted as the effective number of atoms present in the detection beam per unit beam section. This interpretation may be made more precise by rewriting $\sigma$ as:

$$
\sigma=\int \frac{d n(x)}{S(x)}
$$

where $d n(x)$ is the number of atoms present in the detection beam in a thin layer of length $d x$, that is the number obtained by integrating the atomic density $\rho(\mathbf{r})$ over the beam area with a Gaussian weight function $f(\mathbf{r})$ :

$$
\frac{d n(x)}{d x}=\int f(\mathbf{r}) \rho(\mathbf{r}) d y d z
$$


This effective number has to be distinguished from the number of atoms present in the whole cloud in a thin layer of length $d x$, which is obtained by integrating the atomic density $\rho(\mathbf{r})$ :

$$
\frac{d \mathcal{N}(x)}{d x}=\int \rho(\mathbf{r}) d y d z
$$

The atomic medium considered in this paper consists in a freely falling cloud of cold atoms. We will assume that the phase space distribution $\pi(\mathbf{r}, \mathbf{v}, 0)$, at time $t=0$ chosen as the beginning of the free fall, is a Gaussian function of position as well as of velocity:

$$
\pi(\mathbf{r}, \mathbf{v}, 0)=\frac{\mathcal{N}}{\left(2 \pi \sigma_{r} \sigma_{v}\right)^{3}} \exp \left(-\frac{\mathbf{r}^{2}}{2 \sigma_{r}^{2}}-\frac{\mathbf{v}^{2}}{2 \sigma_{v}^{2}}\right)
$$

$\mathcal{N}$ is the total number of atoms while $\sigma_{\mathrm{r}}$ measures the radius of the initial cloud and $\sigma_{v}$ the thermal velocity related to the trap temperature $T$ through:

$$
\sigma_{v}^{2}=\frac{k_{B} T}{m}
$$

As already mentioned in the introduction, this Gaussian approximation gives a rather good description of the trap distribution [2, 7. It presents the great advantage that it will lead to analytical expressions for the integrals encountered in the evaluation of the mean atomic number and of atomic number fluctuations, at least in the case where the Rayleigh divergence length is larger than the cloud size. For the sake of simplicity, the initial cloud has been considered to be centrered on the detection beam, and the detection beam supposed to propagate in horizontal direction. Note however that releasing these two simplifying assumptions would not change the Gaussian character of the atomic density, which is the key point of the following calculations.

The motion of atoms at later times $t$ is determined by the laws of free fall, which preserve the phase space volume:

$$
d \mathbf{r}(t) d \mathbf{v}(t)=d \mathbf{r}(0) d \mathbf{v}(0)
$$

The time-evolution of the phase space distribution $\pi(\mathbf{r}, \mathbf{v}, t)$ is deduced from the conservation of the number of atoms (Liouville's theorem):

$$
\pi\left(\mathbf{r}+\mathbf{v} t+\frac{1}{2} \mathbf{g} t^{2}, \mathbf{v}+\mathbf{g} t, t\right)=\pi(\mathbf{r}, \mathbf{v}, 0)
$$

with $\mathbf{g}$ oriented downwards in the $z$ direction, or equivalently:

$$
\pi(\mathbf{r}, \mathbf{v}, t)=\pi\left(\mathbf{r}-\mathbf{v} t+\frac{1}{2} \mathbf{g} t^{2}, \mathbf{v}-\mathbf{g} t, 0\right)
$$

The atomic density is defined as the integral over velocity of the phase space distribution: 


$$
\rho(\mathbf{r}, t)=\int \pi(\mathbf{r}, \mathbf{v}, t) d \mathbf{v}
$$

This Gaussian integral is easily evaluated [6]:

$$
\rho(\mathbf{r}, t)=\frac{\mathcal{N}}{\left[2 \pi\left(\sigma_{r}^{2}+\sigma_{v}^{2} t^{2}\right)\right]^{3 / 2}} \exp \left[-\frac{\left(\mathbf{r}-\frac{1}{2} \mathbf{g} t^{2}\right)^{2}}{2\left(\sigma_{r}^{2}+\sigma_{v}^{2} t^{2}\right)}\right]
$$

For example, the atomic density at the initial center of the cloud $(\mathbf{r}=\mathbf{0})$ is:

$$
\rho(\mathbf{0}, t)=\frac{\mathcal{N}}{\left[2 \pi \sigma_{r}^{2}\right]^{3 / 2}}\left[\frac{\tau_{r}^{2}}{\tau_{r}^{2}+t^{2}}\right]^{3 / 2} \exp \left[\frac{-t^{4}}{\tau_{g}^{2}\left(\tau_{r}^{2}+t^{2}\right)}\right]
$$

with:

$$
\begin{gathered}
\tau_{r}=\frac{\sigma_{r}}{\sigma_{v}} \\
\tau_{g}=\frac{2 \sqrt{2} \sigma_{v}}{|\mathbf{g}|}
\end{gathered}
$$

This shows that the atomic cloud undergoes a ballistic expansion on a time scale $\tau_{r}$, the time of flight through the trap radius $\sigma_{r}$ for an atom flying at mean thermal velocity $\sigma_{v}$, while at the same time the cloud is falling down under the influence of gravity on a time scale $\tau_{g}$. When the time scale $\tau_{g}$ is longer than $\tau_{r}$, the effect of gravity may be disregarded for short times $\left(t \ll \tau_{g}\right)$ and the atomic density varies mainly as a consequence of ballistic expansion. Gravity leads to an exponential decrease at longer times $\left(t \gg \tau_{g}\right)$ and the variation of the atomic density may be rewritten in this case:

$$
\rho(\mathbf{0}, t) \approx \frac{\mathcal{N}}{\left[2 \pi \sigma_{r}^{2}\right]^{3 / 2}}\left[\frac{\tau_{r}^{2}}{\tau_{r}^{2}+t^{2}}\right]^{3 / 2} \exp \left[-\frac{t^{2}}{\tau_{g}^{2}}\right]
$$

A third time scale $\tau_{w}$ will be found in the following, which will measure the time of flight through the detection beam:

$$
\tau_{w}(x)=\frac{w(x)}{2 \sigma_{v}}
$$

\section{Effective number of atoms deduced from the linear phase shift}

Using the results of the previous section, the linear phase shift of the field due to the interaction with the atomic cloud may be written in terms of equations $(20,23,24)$ where we have introduced a time-dependence of the effective atomic 
number per beam section $\sigma(t)$ in order to account for the quasistatic timevariation of the atomic density $\rho(\mathbf{r}, t)$ :

$$
\begin{gathered}
\frac{d A}{A}=-\alpha_{l} \frac{3 \lambda^{2}}{4 \pi} \sigma(t) \\
\sigma(t)=\int \frac{d n(x, t)}{S(x)} \\
\frac{d n(x, t)}{d x}=\int f(\mathbf{r}) \rho(\mathbf{r}, t) d y d z
\end{gathered}
$$

The latter integral over the transverse variables $y$ and $z$ has a Gaussian character and can be readily performed. The resulting expression for the number of atoms $d n(x, t)$ present at time $t$ in the detection beam in a thin layer of length $d x$ may be written:

$$
d n(x, t)=\frac{d \mathcal{N}(x, t) w^{2}(x)}{4\left(\sigma_{r}^{2}+\sigma_{v}^{2} t^{2}\right)+w^{2}(x)} \exp \left[-\frac{\frac{1}{2} g^{2} t^{4}}{4\left(\sigma_{r}^{2}+\sigma_{v}^{2} t^{2}\right)+w^{2}(x)}\right]
$$

$d \mathcal{N}(x, t)$ is the number of atoms present at time $t$ in the whole cloud in a thin layer of length $d x$ (cf. definition 25):

$$
\frac{d \mathcal{N}(x, t)}{d x}=\frac{\mathcal{N}}{\sqrt{2 \pi\left(\sigma_{r}^{2}+\sigma_{v}^{2} t^{2}\right)}} \exp \left[-\frac{x^{2}}{2\left(\sigma_{r}^{2}+\sigma_{v}^{2} t^{2}\right)}\right]
$$

The integral over the longitudinal variable $x$ keeps a Gaussian character either when the beam size is much smaller than the trap radius $\left(w \ll \sigma_{r}\right)$ or when the Rayleigh divergence length is much larger than the cloud size $\left(\sigma_{r} \ll l_{R}\right)$. We discuss these two limiting cases in the next paragraphs, and recall that both assumptions are fulfilled in the experimental situation of reference [3].

We consider first the limiting case $w \ll \sigma_{r}$, but with $\sigma_{r} / l_{R}$ arbitrary, where the number of atoms $d n(x, t)$ given by equation (41) is proportional to $w^{2}(x)$, and therefore also to the beam section $S(x)$. The $x$-dependent factor $S(x)$ disappears in the expression (39) of $\sigma(t)$ which is thus a Gaussian integral. Its evaluation leads to:

$$
\sigma(t)=\frac{\mathcal{N}}{2 \pi \sigma_{v}^{2}\left(\tau_{r}^{2}+t^{2}\right)} \exp \left[-\frac{t^{4}}{\tau_{g}^{2}\left(\tau_{r}^{2}+t^{2}\right)}\right]
$$

where $\tau_{r}$ and $\tau_{g}$ are the already introduced time scales which correspond respectively to free flight through the trap and free fall (compare with equation 33).

In the case where the Rayleigh divergence length is much larger than the cloud size $\left(\sigma_{r} \ll l_{R}\right.$ with $w / \sigma_{r}$ arbitrary), the $x$-dependence of the beam section $S$ can be ignored and $\sigma(t)$ is again a Gaussian integral. For an arbitrary 
beam size $w$, the time of flight through the probe beam $\tau_{w}$ becomes a relevant parameter. After the evaluation of the integral, we obtain $\sigma(t)$ as:

$$
\sigma(t)=\frac{\mathcal{N}}{2 \pi \sigma_{v}^{2}\left(\tau_{r}^{2}+\tau_{w}^{2}+t^{2}\right)} \exp \left[-\frac{t^{4}}{\tau_{g}^{2}\left(\tau_{r}^{2}+\tau_{w}^{2}+t^{2}\right)}\right]
$$

where $\tau_{w}$ is the time of flight through the probe beam. When both assumptions, a large Rayleigh divergence length and a small beam size $\left(w \ll \sigma_{r}\right.$ and $\left.\sigma_{r} \ll l_{R}\right)$, are valid, expression (44) is identical to expression (43) which is valid for any ratio $\sigma_{r} / l_{R}$.

As already discussed with the help of equation (33), the effect of gravity may be disregarded for times $t \ll \tau_{g}$ when $\tau_{r} \ll \tau_{g}$. It follows that $n(t)$ thus varies as a Lorentzian function of time, as a consequence of ballistic explosion. Gravity leads to an exponential decrease at longer times. In the particular case $\tau_{w} \ll \tau_{r} \ll \tau_{g}$ for example, these various regimes of time-dependence of $\sigma(t)$ may be described in a single formula:

$$
\sigma(t)=\frac{\mathcal{N}}{2 \pi \sigma_{v}^{2}\left(\tau_{r}^{2}+t^{2}\right)} \exp \left[-\frac{t^{2}}{\tau_{g}^{2}}\right]
$$

This condition $\tau_{r} \ll \tau_{g}$ corresponds to a high temperature limit, since it may be rewritten from equations (2734, 35):

$$
k_{B} T \gg \frac{m \sigma_{r}|\mathbf{g}|}{2 \sqrt{2}}
$$

In the opposite low temperature limit, the variation of $\sigma(t)$ is mainly determined for all times by the exponential factor which describes the free fall of the cloud under the effect of gravity.

\section{Effective number of atoms deduced from the nonlinear phase shift}

In the previous section, we have studied the field modification in a linear regime, and deduced an effective number of atoms from a linear phase shift. We now come to the general case where the atomic polarizability may be saturated.

The transverse variation of $d E(\mathbf{r})$ is then completely different from the transverse variation of the incident field $E(\mathbf{r})$ as a consequence of the dependence of the saturation parameter (see equations 12 16):

$$
\begin{gathered}
d E(\mathbf{r})=-\frac{\alpha_{l}}{1+2 s(\mathbf{r})} \frac{3 \lambda^{2}}{4 \pi} \rho(\mathbf{r}) E(\mathbf{r}) d x \\
s(\mathbf{r})=s_{m}(x) f(\mathbf{r})
\end{gathered}
$$

$s_{m}$ is the saturation parameter evaluated on the beam axis: 


$$
s_{m}(x)=\frac{3 \lambda^{2}}{4 \pi S(x)} \frac{|A|^{2}}{\gamma} \frac{1}{1+\delta^{2}}
$$

As in the linear case, we ignore the field radiated into higher-order modes and we obtain the modification of the field amplitude $d A$ projected onto the Gaussian mode through equation (19):

$$
\frac{d A}{A}=-\alpha_{l} \frac{3 \lambda^{2}}{4 \pi} \sigma_{s}(t)
$$

where $\sigma_{s}$ is now modified by saturation:

$$
\begin{gathered}
\sigma_{s}(t)=\int \frac{d n_{s}(x, t)}{S(x)} \\
\frac{d n_{s}(x, t)}{d x}=\int \frac{f(\mathbf{r})}{1+2 s_{m}(x) f(\mathbf{r})} \rho(\mathbf{r}, t) d y d z
\end{gathered}
$$

In order to evaluate $\sigma_{s}$, we will first expand $d n_{s}$ in powers of the saturation parameter:

$$
d n_{s}(x, t)=\sum_{k=0}^{\infty}\left(-2 s_{m}(x)\right)^{k} d n^{(1+k)}(x, t)
$$

where:

$$
\frac{d n^{(j)}(x, t)}{d x}=\int f(\mathbf{r})^{j} \rho(\mathbf{r}, t) d y d z
$$

$d n^{(1)}(x, t)$ is exactly the expression (40) of the effective number of atoms $d n(x, t)$ in the detection beam in a thin layer of length $d x$, as it was evaluated in the previous section. Due to the Gaussian shape of function $f(\mathbf{r})$, function $f(\mathbf{r})^{j}$ has the same expression as $f(\mathbf{r})$ with a modified value of the beam size parameter, precisely with $w^{2}(x)$ replaced by $\left(w^{2}(x) / j\right)$. Therefore, $d n^{(j)}(x, t)$ corresponds to the expression (41) of $d n(x, t)$ evaluated for a modified beam size:

$$
d n^{(j)}(x, t)=\frac{d \mathcal{N}(x, t) \frac{w^{2}(x)}{j}}{4\left(\sigma_{r}^{2}+\sigma_{v}^{2} t^{2}\right)+\frac{w^{2}(x)}{j}} \exp \left[-\frac{\frac{1}{2} g^{2} t^{4}}{4\left(\sigma_{r}^{2}+\sigma_{v}^{2} t^{2}\right)+\frac{w^{2}(x)}{j}}\right]
$$

These expressions are greatly simplified in the limiting case of a small beam size $\left(w \ll \sigma_{r}\right)$, where $d n^{(j)}(x, t)$ is proportional to $w^{2}(x)$, so that:

$$
d n^{(j)}(x, t)=\frac{d n(x, t)}{j}
$$


Assuming furthermore that the Rayleigh divergence length is large $\left(\sigma_{r} \ll l_{R}\right)$, one may disregard the $x$-dependence of the on-axis saturation parameter $s_{m}$ and obtain a closed analytical expression for $d n_{s}(x, t)$ and therefore for $\sigma_{s}$ :

$$
\sigma_{s}(t)=\frac{\sigma(t)}{2 s_{m}} \ln \left(1+2 s_{m}\right)
$$

This expression may then be used to derive the bistability relation which connects the mean field sent into the cavity and the mean field inside the cavity (see for example 9]). An important consequence of this equation is that $\sigma_{s}(t)$ and $\sigma(t)$ have exactly the same time dependence. It follows that the evaluations of the number of atoms obtained from the linear and non linear phase shift agree as soon as the transverse structure of the mode has been properly accounted for. It is however worth to stress that this property relies on the two simplifying assumptions $w \ll \sigma_{r}$ and $\sigma_{r} \ll l_{R}$ which have been used to derive equation (57), and does no longer hold in a more general situation.

\section{Fluctuations of the number of atoms}

Up to now, we have studied only the mean value of the field modification in the atomic medium. We now want to evaluate the fluctuations of this modification which are associated with fluctuations of the number of atoms coupled to the field. As shown by the previous discussions, the definition of such a number is not simple in the general case. This is why we will study the fluctuations of the number of atoms with the help of simplifying assumptions.

First, we will restrict our attention to the regime of a linear polarisability where the field modification is related to the quantity $\sigma(t)$ given by equation (23). We are indeed interested mainly in fluctuations of the linear phase shift, which is usually much larger than the nonlinear one. We will then consider that the Rayleigh divergence length is much larger than the cloud size $\left(\sigma_{r} \ll l_{R}\right)$, so

that the beam area $S$ is independent of $x$ and equation (23) may be rewritten in terms of an effective number $n(t)$ of atoms in the probe beam at time $t$ :

$$
\sigma(t)=\frac{n(t)}{S}
$$

with:

$$
n(t)=\int d n(x, t)=\int f(\mathbf{r}) \rho(\mathbf{r}, t) d \mathbf{r}
$$

It would be possible to study the fluctuations of $\sigma(t)$ in the general case, using the same techniques as presented below. However, it will be more instructive to discuss the fluctuations of $n(t)$, which is a dimensionless number and is expected to have a nearly Poissonian statistics. For completeness, note that in the limiting 
case studied in the present section $\left(\sigma_{r} \ll l_{R}\right), n(t)$ is simply the product of $\sigma(t)$ by the beam section $S$.

We come now to the discussion of atomic number fluctuations. To this aim, we notice that the atomic density $\rho(\mathbf{r}, t)$ may be considered as an average value taken over the random distribution of the atomic positions, precisely over the random values of the initial positions and velocities:

$$
\begin{gathered}
\rho(\mathbf{r}, t)=\langle P(\mathbf{r}, t)\rangle \\
P(\mathbf{r}, t)=\sum_{i=1}^{\mathcal{N}} \delta\left(\mathbf{r}-\mathbf{r}_{i}(t)\right)=\sum_{i=1}^{\mathcal{N}} \delta\left(\mathbf{r}-\mathbf{r}_{i}(0)-\mathbf{v}_{i}(0) t-\frac{1}{2} \mathbf{g} t^{2}\right)
\end{gathered}
$$

where $i$ labels the atoms $(i=1 \ldots \mathcal{N}$ with a total number of atoms $\mathcal{N}$ in the cloud); $\mathbf{r}_{i}(t)$ is the position of the $i$ th atom at time $t ; \mathbf{r}_{i}(0)$ and $\mathbf{v}_{i}(0)$ are its initial position and velocity. Therefore, $n(t)$ may be considered as the mean value of a random number $N(t)$ :

$$
N(t)=\sum_{i=1} \mathcal{N} f\left(\mathbf{r}_{i}(t)\right)
$$

This random number has fluctuations which, as already discussed in the introduction, may reveal themselves as fluctuations of the linear phase shift and then of the cavity-detuning parameter.

In order to discuss the statistical properties of these numbers, we will consider that the atoms are statistically uncorrelated in the initial distribution. We will define covariances of random variables according to the general prescription:

$$
\langle u, v\rangle=\langle u v\rangle-\langle u\rangle\langle v\rangle
$$

$d \mathcal{N}_{0}$ is the random variable associated with the number of atoms in an elementary volume $d \mathbf{r} d \mathbf{v}$ of the phase space at time $t=0$. We consider it to be a Poisson random variable:

$$
\left\langle d \mathcal{N}_{0}, d \mathcal{N}_{0}\right\rangle=\left\langle d \mathcal{N}_{0}\right\rangle=\pi(\mathbf{r}, \mathbf{v}, 0) d \mathbf{r} d \mathbf{v}
$$

while different $d \mathcal{N}_{0}$ and $d \mathcal{N}_{0}^{\prime}$ evaluated for non overlapping elementary volumes are uncorrelated random variables:

$$
\left\langle d \mathcal{N}_{0}, d \mathcal{N}_{0}^{\prime}\right\rangle=0
$$

Before coming to the discussion of the fluctuations of the effective atomic number in the detection beam, we want to recall briefly how these assumptions are commonly used to derive Poisson statistics. Usually, one studies a number of atoms integrated in a finite volume: 


$$
N_{0}=\int d \mathcal{N}_{0} f_{v}(\mathbf{r})
$$

where the function $f_{v}$ is either 1 inside the detection volume or 0 otherwise; this function therefore obeys the property $f_{v}^{2}=f_{v}$. By summing up the variances corresponding to the various elementary volumes, one deduces that the integrated number $N_{0}$ has a Poissonian variance:

$$
\left\langle N_{0}, N_{0}\right\rangle=\int\left\langle d \mathcal{N}_{0}\right\rangle f_{v}^{2}(\mathbf{r})=\left\langle N_{0}\right\rangle
$$

The same conclusion is reached for the number of atoms integrated in a finite volume after a free fall during a time $t$ :

$$
\begin{gathered}
N(t)=\int d \mathcal{N}_{0} f_{v}\left(\mathbf{r}+\mathbf{v} t+\frac{1}{2} \mathbf{g} t^{2}\right) \\
\langle N(t), N(t)\rangle=\int\left\langle d \mathcal{N}_{0}\right\rangle f_{v}^{2}\left(\mathbf{r}+\mathbf{v} t+\frac{1}{2} \mathbf{g} t^{2}\right)=\langle N(t)\rangle
\end{gathered}
$$

The case studied in the present paper does not lead to such a simple conclusion since the effective number is defined with a Gaussian weight function which does not obey the property $f^{2}=f$. By summing up the variances corresponding to the various elementary volumes, one obtains the variance of this effective number to be:

$$
\langle N(t), N(t)\rangle=\int f^{2}\left(\mathbf{r}+\mathbf{v} t+\frac{1}{2} \mathbf{g} t^{2}\right) \pi(\mathbf{r}, \mathbf{v}, 0) d \mathbf{r} d \mathbf{v}
$$

By changing variables from $\{\mathbf{r}(0), \mathbf{v}(0)\}$ to $\{\mathbf{r}(t), \mathbf{v}(t)\}$ and integrating over the velocity distribution, this variance can be rewritten as:

$$
\langle N(t), N(t)\rangle=\int f^{2}(\mathbf{r}) \rho(\mathbf{r}, t) d \mathbf{r}
$$

while the mean value is:

$$
\langle N(t)\rangle=\int f(\mathbf{r}) \rho(\mathbf{r}, t) d \mathbf{r}
$$

The mean value is obtained as the product of the expression (44) evaluated previously for $\sigma(t)$ with the effective beam section $\left(S=\pi w^{2} / 2\right)$ :

$$
\langle N(t)\rangle=\frac{\mathcal{N} \tau_{w}^{2}}{\tau_{r}^{2}+\tau_{w}^{2}+t^{2}} \exp \left[-\frac{t^{4}}{\tau_{g}^{2}\left(\tau_{r}^{2}+\tau_{w}^{2}+t^{2}\right)}\right]
$$

The variance has the same expression evaluated for a modified beam size parameter $\left(w^{2}\right.$ replaced by $\left(w^{2} / 2\right)$, i.e. $\tau_{w}^{2}$ replaced by $\left.\left(\tau_{w}^{2} / 2\right)\right)$ : 


$$
\langle N(t), N(t)\rangle=\frac{\mathcal{N} \tau_{w}^{2}}{2 \tau_{r}^{2}+\tau_{w}^{2}+2 t^{2}} \exp \left[-\frac{2 t^{4}}{\tau_{g}^{2}\left(2 \tau_{r}^{2}+\tau_{w}^{2}+2 t^{2}\right)}\right]
$$

The variance is therefore smaller than the mean value, which implies that the distribution of the random variable $N(t)$ has always a sub-Poissonian character:

$$
\langle N(t), N(t)\rangle<\langle N(t)\rangle
$$

In the limiting case where the detection beam waist $w$ is much smaller than the trap radius $\sigma_{r}$, the variance is simply half the mean value:

$$
\langle N(t), N(t)\rangle=\frac{1}{2}\langle N(t)\rangle
$$

We now look for a dynamical characterization of temporal correlations rather than for a static statistical description. Precisely, we wish to evaluate the covariance function for the numbers of atoms $N(t)$ and $N(t \prime)$ present in the detection beam at two different times. By summing up the variances corresponding to the various elementary volumes, one obtains this covariance function as:

$$
\left\langle N(t), N\left(t^{\prime}\right)\right\rangle=\int f\left(\mathbf{r}+\mathbf{v} t+\frac{1}{2} \mathbf{g} t^{2}\right) f\left(\mathbf{r}+\mathbf{v} t^{\prime}+\frac{1}{2} \mathbf{g} t^{\prime 2}\right) \pi(\mathbf{r}, \mathbf{v}, 0) d \mathbf{r} d \mathbf{v}
$$

Since the positions at times $t$ and $t^{\prime}$ are linear functions of the initial position and initial velocity, this expression appears as a six-dimensional Gaussian integral which may be readily evaluated. We will write the covariance function:

$$
C_{N N}(T, \tau)=\left\langle N(t), N\left(t^{\prime}\right)\right\rangle
$$

as a function of the mean time $T$ and of the delay $\tau$ between the two time parameters $t$ and $t^{\prime}$ :

$$
T=\frac{t+t^{\prime}}{2} \quad \tau=t-t^{\prime}
$$

The time $T$ will be associated with the global time variation of the atomic number during the free fall of the cloud while the delay $\tau$ will rather correspond to the correlation between numbers evaluated at different times.

The explicit evaluation of the Gaussian integral (77) gives the following expression:

$$
C_{N N}(T, \tau)=n(0) L(T, \tau) \exp [-M(T, \tau)]
$$

where:

$$
n(0)=\frac{\mathcal{N} \tau_{w}^{2}}{\tau_{r}^{2}+\tau_{w}^{2}}
$$




$$
\begin{gathered}
L(T, \tau)=\frac{\tau_{w}^{2}\left(\tau_{r}^{2}+\tau_{w}^{2}\right)}{2 \tau_{w}^{2} T^{2}+\left(\tau_{r}^{2}+\frac{\tau_{w}^{2}}{2}\right)\left(\tau^{2}+2 \tau_{w}^{2}\right)} \\
M(T, \tau)=\frac{\left(T^{2}+\frac{\tau^{2}}{4}\right)^{2}\left(\tau^{2}+2 \tau_{w}^{2}\right)+4\left(\tau_{r}^{2}+\frac{\tau_{w}^{2}}{2}\right) T^{2} \tau^{2}}{\tau_{g}^{2}\left[2 \tau_{w}^{2} T^{2}+\left(\tau_{r}^{2}+\frac{\tau_{w}^{2}}{2}\right)\left(\tau^{2}+2 \tau_{w}^{2}\right)\right]}
\end{gathered}
$$

The expression of the variance of the number $N(T)$ evaluated at a given time $T$ is recovered at $\tau=0$ :

$$
C_{N N}(T, 0)=\langle N(T), N(T)\rangle
$$

Instead evaluating the exact expressions (80 83), we will rather concentrate on the limiting case where the detection waist is much smaller than the trap radius. This assumption implies in particular that the correlation time $\tau_{w}$ is much smaller than the global fall time $\tau_{r}$. This will allow us to treat the number of atoms as a quasistationary random variable. Considering only not too long delays $\tau \ll \tau_{r}$ and not too short fall times $T \gg \tau_{w}$, we may rewrite the covariance function in terms of two scaled time parameters $T / \tau_{r}$ and $\tau / \tau_{w}$ :

$$
C_{N N}(T, \tau)=n(0) L(T, \tau) \exp \left[-\zeta\left(a_{T}-b_{T} L(T, \tau)\right)\right]
$$

with:

$$
\begin{gathered}
n(0)=\frac{\mathcal{N} \tau_{w}^{2}}{\tau_{r}^{2}} \\
L(T, \tau)=\frac{1}{\left(\frac{\tau}{\tau_{w}}\right)^{2}+\alpha_{T}^{2}} \\
\alpha_{T}^{2}=2\left[1+\left(\frac{T}{\tau_{r}}\right)^{2}\right] \\
a_{T}=\left(\frac{T}{\tau_{r}}\right)^{2}\left[4+\left(\frac{T}{\tau_{r}}\right)^{2}\right] \\
b_{T}=2\left(\frac{T}{\tau_{r}}\right)^{2}\left[2+\left(\frac{T}{\tau_{r}}\right)^{2}\right]^{2} \\
\zeta=\frac{\tau_{r}^{2}}{\tau_{g}^{2}}
\end{gathered}
$$

In the limiting case $\zeta \ll 1$ where the time scale characteristic of gravity is long, the covariance function is essentially a Lorentzian function. More generally when 
including the effect of gravity, it may be expanded in terms of powers of this Lorentzian function:

$$
C_{N N}(T, \tau)=n(0) e^{-\zeta a_{T}} \sum_{k=0}^{\infty}\left(\zeta b_{T}\right)^{k} \frac{L^{1+k}}{k !}
$$

Since the correlation time, of the order of $\tau_{w}$, is much smaller than the characteristic time of variation of the mean values, of the order of $\tau_{r}$, the number of detected atoms may be considered as a quasistationary random variable and it is therefore possible to characterize its fluctuations by a noise spectrum $S_{N N}(T, \omega)$ :

$$
S_{N N}(T, \omega)=\int d \tau \quad e^{i \omega \tau} C_{N N}(T, \tau)
$$

This spectrum is obtained through a Fourier transformation with respect to the delay $\tau$ and it slowly depends upon the time of fall $T$. It has the same definition as the ambiguity function of radar theory 10 which is the analog for time-frequency distributions of the Wigner distribution for position-momentum distributions [11]. Because of the quasistationary character of the random variable $N(t)$, it will remain positive for all values of the parameters, as the noise spectrum for a stationary random variable. The spectrum may be written as the product of the variance at time $T$ by a normalized spectrum:

$$
\begin{gathered}
S_{N N}(T, \omega)=C_{N N}(T, 0) \bar{S}(T, \omega)=\frac{1}{2} n(T) \bar{S}_{N N}(T, \omega) \\
\int \frac{d \omega}{2 \pi} \bar{S}_{N N}(T, \omega)=1
\end{gathered}
$$

In the limiting case $\zeta \ll 1$, the effect of gravity upon the correlation function $C_{N N}(T, \tau)$ may be disregarded, so that the latter is a Lorentzian function of $\tau$ and the noise spectrum has an exponential shape:

$$
S_{N N}(T, \omega)=n(0) \frac{\pi \tau_{w}}{\alpha_{T}} e^{-\alpha_{T} \omega \tau_{w}}
$$

The normalized spectrum:

$$
\bar{S}_{N N}(T, \omega)=\pi \alpha_{T} \tau_{w} e^{-\alpha_{T} \omega \tau_{w}}
$$

has a linewidth $\left(\alpha_{T} \tau_{w}\right)^{-1}$ and a peak value $\left(\pi \alpha_{T} \tau_{w}\right)$ mainly determined by the time of flight $\tau_{w}$ through the probe beam.

In the general case of an arbitrary $\zeta$, the noise spectrum may be deduced from expression (92) of the correlation function (using formulas 8.432.5 and 8.468 in ref. [12]) as: 


$$
S_{N N}(T, \omega)=n(0) \frac{\pi \tau_{w}}{\alpha_{T}} e^{-\alpha_{T} \omega \tau_{w}} e^{-\zeta a_{T}} \sum_{k=0}^{\infty}\left(\frac{\zeta b_{T}}{4}\right)^{k} \frac{p_{k}\left(\alpha_{T} \omega \tau_{w}\right)}{(k !)^{2}}
$$

where $p_{k}$ is a polynomial function of frequency:

$$
p_{k}(x)=\sum_{j=0}^{k} \frac{(2 x)^{j}(2 k-j) !}{j !(k-j) !}
$$

This leads to a normalized spectrum:

$$
\bar{S}_{N N}(T, \omega)=\pi \alpha_{T} \tau_{w} e^{-\alpha_{T} \omega \tau_{w}} e^{-\frac{\zeta b_{T}}{\alpha_{T}^{2}}} \sum_{k=0}^{\infty}\left(\frac{\zeta b_{T}}{4}\right)^{k} \frac{p_{k}\left(\alpha_{T} \omega \tau_{w}\right)}{(k !)^{2}}
$$

with a peak value at zero frequency:

$$
\bar{S}_{N N}(T, 0)=\pi \alpha_{T} \tau_{w} e^{-\frac{\zeta b_{T}}{\alpha_{T}^{2}}} \sum_{k=0}^{\infty}\left(\frac{\zeta b_{T}}{4}\right)^{k} \frac{(2 k) !}{(k !)^{3}}
$$

The expressions (98 101) provide a quantitative evaluation of the fluctuations of the number of atoms coupled to the probe beam in the general case of a motion under gravity. They may be used for assessing the effect of these number fluctuations in any high precision measurements such as spectroscopic, nonlinear or quantum optics experiments where the falling cloud acts as the atomic medium. For only qualitative assessments, we can draw the following two conclusions: first, the instantaneous variance of the effective atomic number has a subPoissonian character; it is precisely half the Poissonian variance at the limit of a small probe beam size. Second, the correlation time for the fluctuations of the effective atomic number is mainly determined by the time of flight $\tau_{w}$ through the probe beam.

\section{Fluctuations of the cavity detuning}

We now focus our attention on experiments where the probe field goes through an optical cavity containing the cloud of cold atoms. As already discussed in the introduction, atomic number fluctuations emulate fluctuations of the cavity detuning. The present section is devoted to a precise evaluation of this effect. For simplicity, we consider only the regime of a linear polarisability in the limit where the Rayleigh divergence length is much larger than the cloud size (same simplifying assumptions as in section 5 ). We also consider the dispersive regime where the atom is excited far from resonance $\left(\delta^{2} \gg 1\right)$. 
A key parameter in the experiments with atoms inside a cavity is the socalled cooperativity parameter (see for example [13, 14] and references therein):

$$
C=\frac{3 \lambda^{2}}{4 \pi S} \frac{n}{2 \kappa \tau_{c}}
$$

where $\kappa$ is the decay rate of the intracavity field and $\tau_{c}$ the cavity round-trip time $\left(2 \kappa \tau_{c}\right.$ is just the intensity transmission of the coupling mirror), while $S$ and $n$ are respectively the beam section and the effective atomic number studied in the present paper. The effect of the atomic medium on the field may be written as a variation $\Phi$ of the cavity detuning (see for example [14]):

$$
\Phi=\frac{2 \kappa C}{\delta}=\frac{3 \lambda^{2}}{4 \pi S} \frac{n}{\delta \tau_{c}}
$$

The fluctuations of the atomic number $N$ studied in the previous section are therefore equivalent to fluctuations of the cavity detuning $\Phi$ characterized by a noise spectrum:

$$
S_{\Phi \Phi}(T, \omega)=\left(\frac{3 \lambda^{2}}{4 \pi S}\right)^{2} \frac{S_{N N}(T, \omega)}{\left(\delta \tau_{c}\right)^{2}}
$$

that is (using equations 94 and 102):

$$
S_{\Phi \Phi}(T, \omega)=\kappa \frac{C(T)}{\delta^{2}} \frac{3 \lambda^{2}}{4 \pi S} \frac{\bar{S}_{N N}(T, \omega)}{\tau_{c}}
$$

We have introduced here a time-dependent cooperativity parameter $C(T)$ proportional to the mean effective number $n(T)$ at time $T$. Since the cavity detuning $\Phi$ is a frequency, its noise spectrum $S_{\Phi \Phi}$ also has the dimension of a frequency. The first factor appearing in its expression (105) is the frequency $\kappa$, which can be regarded as measuring the noise spectrum of detuning fluctuations associated with the decay of the intracavity field through the coupling mirror. The other factors which appear in equation (105) have been written in dimensionless terms.

As long as $S_{\Phi \Phi}$ remains smaller than $\kappa$, the effect of the detuning fluctuations on cavity dynamics remains in a linear regime. In the opposite case, it would be necessary to give a more elaborate treatment of these fluctuations accounting for multiplicative noise processing. It is worth stressing that detuning fluctuations may not be considered as a white noise, since the spectrum has a width essentially given by the inverse of the time of flight through the detection beam.

In the case where the measurements are performed at frequencies much higher than this spectral width, the detuning fluctuations become negligible. This is in particular the case in the experiment on squeezing with cold atoms

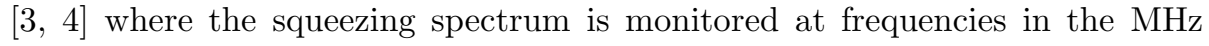


range. It follows that the effective atomic number may be considered as a fluctuationless classical variable, even when it varies as a function of time while the cloud is exploding and falling down. The fluctuations of the effective number might be detectable at much lower frequencies in the $\mathrm{kHz}$ range. In order to detect these fluctuations, it would thus be necessary to get rid of the large excess noise present in this frequency range.

In conclusion, we have investigated the problem of evaluating the number of atoms effectively interacting with a probe beam passing through a cloud of cold atoms released from a magneto-optical trap, accounting for the transverse Gaussian profile of the probe beam, in the general case of a motion under gravity.

We have shown that this problem requires a detailed treatment which depends on the quantity of interest in the specific measurement. We have given explicit evaluations of the effective atomic number for the two cases where the linear or nonlinear phaseshifts are measured. These two cases correspond to a same time variation of the effective number when the two simplifying assumptions of a small beam waist $\left(w \ll \sigma_{r}\right)$ and of a long Rayleigh divergence length $\left(\sigma_{r} \ll l_{R}\right)$ are fulfilled. The more general situation may also be dealt with by using the expressions obtained in sections 3 and 4 .

We have also calculated the correlation functions characterizing the fluctuations of the effective atomic number, focussing attention upon fluctuations of the linear phaseshift since they may be quite large and consequently affect high precision measurements such as spectroscopic, nonlinear or quantum optics experiments. We have found that the instantaneous variance of the effective atomic number has a subPoissonian character, due to the Gaussian profile of the probe beam. In particular, the variance is half the Poissonian variance at the limit of a small beam waist $\left(w \ll \sigma_{r}\right)$. At the same limit, the spectral width characteristic of these fluctuations is essentially the inverse of the time of flight through the probe beam for an atom flying at the mean thermal velocity. The effect of these number fluctuations on the field fluctuations is thus confined to a low frequency range, especially for cold atoms which correspond to relatively long time of flight. For atoms at room temperature, the spectral width would be larger. In both cases, the techniques used in the present paper allow to obtain quantitative estimates of the influence of atomic number fluctuations on field fluctuations, and then to the sensitivity of optical measurements.

Acknowledgements

Thanks are due to Jean Dalibard and Claude Fabre for discussions.

\section{References}

[1] Arimondo E., Phillips W. and Strumia F. eds, Laser Manipulation of Atoms (North Holland, 1992) 
[2] Drewsen M., Laurent Ph., Nadir A., Santarelli G., Clairon A., Castin Y., Grison D. and Salomon C., Applied Physics B59 283 (1994)

[3] Lambrecht A., Courty J.M., Reynaud S. and Giacobino E., Applied Physics B60 129 (1995)

[4] Lambrecht A. et al, Squeezing with cold atoms, preprint (1995)

[5] Schleich W. and Scully M.O., Physical Review A37 1261 (1988)

[6] Weiss D.S. Riis E., Shevy Y., Ungar P.J. and Chu S., Journal of the Optical Society of America B6 2072 (1989)

[7] Ketterle W., Davis K.B., Joffe M.A., Martin A. and Pritchard D.E., Physical Review Letters 702253 (1993)

[8] Heidmann A. and Reynaud S., Journal de Physique 461937 (1985)

[9] Drummond P.D., IEEE Journal of Quantum Electronics QE17 301 (1981)

[10] Papoulis A., Journal of the Optical Society of America 64779 (1974)

[11] Haken H., Review of Modern Physics 4767 (1975)

[12] Gradshteyn I.S. and Ryzhik I.M., Table of integrals, series and products (Academic Press, 1980)

[13] Lugiato L.A., in Progress in Optics XXI 71 (1984)

[14] Hilico L., Fabre C., Giacobino E. and Reynaud S., Physical Review A46 $4397(1992)$ 